\section{Strongly Correlated Electronic Materials: Present and Future}

\section{E. Dagotto and Y. Tokura}

\begin{abstract}
In complex transition-metal oxides, the interactions between the electronic spins, charges, and orbitals produce a rich variety of electronic phases. The competition and/or cooperation among these correlated-electron phases can lead to the emergence of surprising electronic phenomena and functionalities and form the basis for a new type of electronics.
\end{abstract}

widely used path to extract information about models, and this avenue has already led to considerable progress in this area of research.

The importance of understanding how complex phenomena emerge from simple ingredients was identified as one of the challenges for the next decade in the recent study by the U.S. National Academy of Sciences on the current status of condensed-matter and materials physics. ${ }^{4}$ Also, the Basic Energy Sciences Advisory Committee of the U.S. Department of Energy has identified five "grand challenges" for science to reach the ability to control matter all the way to the atomic and electronic levels. ${ }^{5}$ Among these challenges is an understanding of how the remarkable properties of matter emerge from the complex correlations of the atomic or electronic constituents. Without a doubt, controlling correlated electrons is a crucial area of research in condensed-matter and materials science.

This brief article is intended to provide an overview of correlated electrons, with a focus on transition-metal oxides. Our opinions of the present status of the field and its future are also included. This is certainly not a comprehensive review, and interested readers are referred to the articles cited herein for a more detailed perspective and a more complete list of references.

\section{Control of Correlated Electrons in Complex Oxides}

The fundamental parameters for controlling the behavior of correlated electrons are (1) the tunneling electron hopping amplitude $t$ (or the one-electron bandwidth $W$ ) and (2) the density of charge carriers (to be more precise, the band filling). ${ }^{6}$ The hopping amplitude $t$ competes with the on-site electronelectron Coulomb repulsion energy $U$ and the outcome of this competition is the Mott transition, namely, an insulator-metal transition that occurs in correlated-electron systems. As a function of $U / t$, the system undergoes several changes in spin and charge dynamics. In the limit of large $U / t$, every electron localizes on an atomic site when the number of electrons precisely equals the number of atomic sites. In a Mott insulator, the ground state is usually antiferromagnetic in nature. When $U / t$ decreases and reaches some critical value, the transition to the metallic state occurs, as schematically shown in Figure 1. Another route to the Mott transition is by changing the filling (charge doping) of the correlated Mott insulator. Ideally, a minute deviation from half-filling (or from an integer number of 


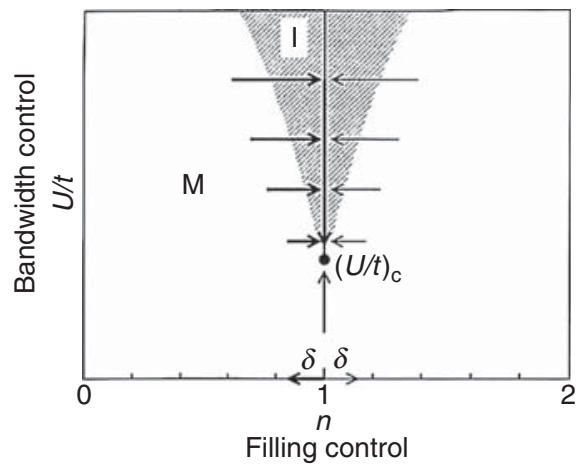

Figure 1. Insulator (I)-metal (M) transitions in correlated-electron system controlled by the bandwidth $W$ (or equivalently by the hopping amplitude $t)$ or the band filling $n$ (doping $x$ ). $U$ is the on-site Coulomb repulsion interaction, and $(U / t)_{\mathrm{c}}$ represents the critical value at which the transition occurs. Note: $\delta=1-n$ for $n<1 ; \delta=n-1$ for $n>1$.
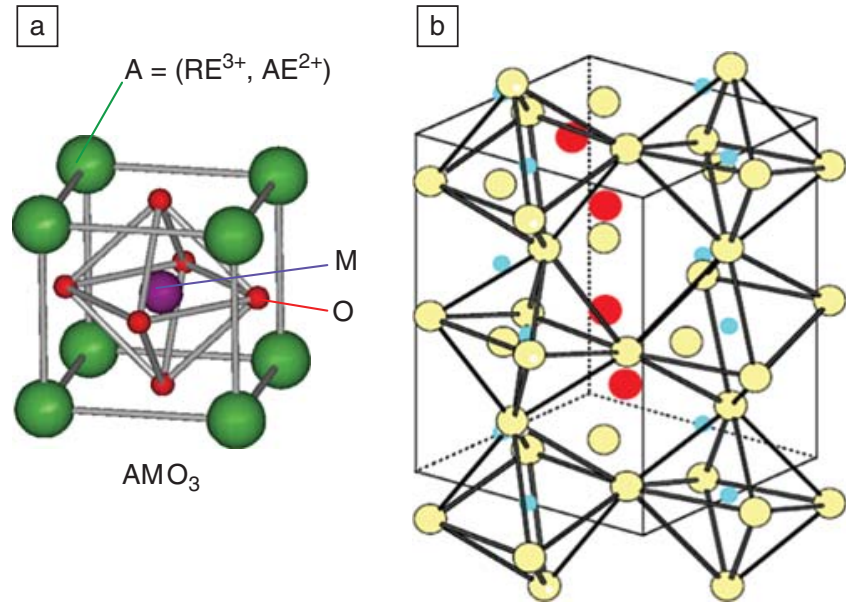

Figure 2. (a) Perovskite structure, $(\mathrm{RE}, \mathrm{AE}) \mathrm{MO}_{3}$, with trivalent (3+) rare-earth $(\mathrm{RE})$ ions and divalent $(2+)$ alkaline-earth $(\mathrm{AE})$ ions at the perovskite $A$ site and the transition-metal element on the perovskite $\mathrm{B}$ site. (b) Orthorhombically distorted $\left(\mathrm{GdFeO}_{3}\right.$-type) structure of perovskites.

erned by the $d$-electron hybridization with the intervening $\mathrm{O} 2 p$ state. The impact of the variation in $W$ with $r_{A}$ is observed, for example, in the metal-insulator transition in the family $\mathrm{RENiO}_{3},{ }^{7} \mathrm{LaNiO}_{3}$, with a maximum tolerance factor $(f \approx 0.96)$ close to unity, is a paramagnetic metal with one conduction $d$ electron per $\mathrm{Ni}$ atom, whereas other $\mathrm{RENiO}_{3}$ materials with smaller $f$ values (or decreasing RE ionic size) exhibit antiferromagnetic insulating ground states and undergo a thermally induced insulator-metal transition (IMT) with increasing temperature.

Another important advantage of perovskites or related structures (e.g., layered structures, termed the RuddlesdenPopper series) is the ease of chemical control of the band filling. Using the solid solution $\mathrm{RE}_{1-x} \mathrm{AE}_{x}$ at the perovskite $A$ site $\left(\mathrm{AMO}_{3}\right.$, see Figure 2a), the effective valence of the transition metal (M) becomes $3+x$. In analogy to doped semiconductors, the increase (decrease) of $x$ is customarily called "hole doping" ("electron doping"). In reality, this change in $x$ reflects a decrease (increase) of the band filling or of the chemical potential.

The most well-known example of a band-filling- (doping-) controlled Mott transition is the case of high-temperature superconductors of copper oxides, such as $\mathrm{La}_{2-x} \mathrm{Sr}_{x} \mathrm{CuO}_{4}$, in which the effective $\mathrm{Cu}^{2+}$ valence state, or more correctly the $[\mathrm{Cu}-\mathrm{O}]^{0}$ state, is doped with holes and becomes a $\mathrm{Cu}^{(2+x)+}\left([\mathrm{Cu}-\mathrm{O}]^{x+}\right)$ state. It is well known that, upon the IMT around $x=0.06$, the ground state becomes superconducting. Another prototypical example is $\mathrm{La}_{1-x} \mathrm{Sr}_{x} \mathrm{TiO}_{3}$, where hole doping of $x \approx 0.05$ drives the ground state from an antiferromagnetic Mott insulator to a paramagnetic metal with a high carrier density (band filling $n=1-x$ ) but with a large enhancement of the carrier mass, as compared to the calculated band mass. ${ }^{8}$

Figure 3 presents a schematic of the overall electronic phase diagram of $\mathrm{RE}_{1-x} \mathrm{AE}_{x} \mathrm{TiO}_{3}$ under the control of both the one-electron bandwidth $W$ and the band filling (i.e., $1-x$ ) in terms of varying $\mathrm{RE}$ ionic radius and doping level $x$. The "undoped" Mott insulator, for example, $\mathrm{La}_{1-y} \mathrm{Y}_{y} \mathrm{TiO}_{3}$, shows an increasing charge gap (Mott-Hubbard gap) with decreasing $W$ (or increasing $U / W$ ), whereas the antiferromagnetic order turns to ferromagnetic through the influence of orbital ordering (see the next section). The critical doping level of the IMT is increased from $x_{c} \approx 0.05$ for $\mathrm{La}_{1-x} \mathrm{Sr}_{x} \mathrm{TiO}_{3}$ with the maximum $W$ value $\left(W_{\mathrm{La}}\right)$, up to $x_{c} \approx 0.4$ for $\mathrm{Y}_{1-x} \mathrm{Ca}_{x} \mathrm{TiO}_{3}$ with a relatively small $\mathrm{W}$ value (about $80 \%$ of $W_{\mathrm{La}}$ ). The rather high hole concentration needed to realize the metallicity of the small- $W$ systems, such as $\mathrm{Y}_{1-x} \mathrm{Ca}_{x} \mathrm{TiO}_{3}$, is quite common in the $3 d$ transition-metal oxides with perovskitelike structures; there should be shortrange/long-range polaronic ordering in such a highly doped insulating ground state because of the combined effect of strong electron correlation and electronlattice coupling. The charge-orbitalordered insulating state in the perovskite manganites is one such example where the state changes substantially when subjected to an external stimulus such as a magnetic field, electric field, or light irradiation, as described in the following sections. 


\section{Ordering of Charge, Spin, and Orbital Degrees of Freedom}

Consider a transition-metal ion $(M)$ in a crystal with perovskite structure. $M$ is surrounded by six doubly negative oxygen ions $\left(\mathrm{O}^{2-}\right)$ that give rise to the crystal field potential and partly lift the degeneracy of the $d$-electron levels (Figure 4). Wave functions pointing toward the $\mathrm{O}^{2-}$ ions $\left(d_{x^{2}-y^{2}}\right.$ and $d_{3 z^{2}-r^{2}}$, called eg orbitals) have higher energy than those pointing between the ions $\left(d_{x y}, d_{y z}\right.$, and $d_{z x}$, called $t_{2 \mathrm{~g}}$ orbitals). In the Mott insulating state, all of the $d$ electrons are localized almost on their respective atoms, making the spin and orbital degrees of freedom simultaneously active. The combination of these degrees of freedom produces versatile spin-orbital ordering patterns. Prototypical cases are shown in Figure 5 for perovskites of $\mathrm{LaVO}_{3}$ and $\mathrm{YVO}_{3}$, which are $t_{2 \mathrm{~g}}$ electron systems, and $\mathrm{LaMnO}_{3}$ and $\mathrm{BiMnO}_{3}$, which are eg electron systems.

In $\mathrm{LaVO}_{3}$, for example, the antiferromagnetic spin ordering with ferromagnetic chains along the $z$ direction is known to induce an orbital-ordered state with alternate occupancy of $d_{y z}$ and $d_{z x}$ in the $x$, $y$, and $z$ directions, in addition to the commonly occupied $d_{x y}$ orbital. $^{9}$ (In Figure 5a, the $d_{x y}$ orbital on each $\mathrm{V}$ site is omitted for clarity.) Despite its nearly cubic character, the electronic structure is highly anisotropic because of the spin-orbital ordering. On the other hand, in $\mathrm{YVO}_{3}$ (Figure 5b), with a larger orthorhombic $\left(\mathrm{GdFeO}_{3}\right.$-type) lattice distortion, staggered spin order and a $d_{y z}$ or $d_{z x}$ orbitalordered state along the $z$ axis are known to exist in the ground state. Thus, the relationships between the spin and orbital orders for these two compounds are exactly opposite, indicating the strong coupling between the spin and orbital degrees of freedom.

In the perovskite manganites, the orbital degeneracy is easily lifted by coupling with the local deformation of the $\mathrm{MnO}_{6}$ octahedron (called the Jahn-Teller effect), such as the elongation and compression of the octahedron along the $z$ axis favoring the occupation of the $d_{3 z^{2}-r^{2}}$ and $d_{x^{2}-y^{2}}$ orbitals, respectively. Therefore, the orbital ordering coupled with the collective Jahn-Teller distortion first emerges with decreasing temperature, and then regulates the spin ordering pattern at lower temperatures. In $\mathrm{LaMnO}_{3}$, a local linear combination of the $d_{3 z^{2}-r^{2}}$ and $d_{x^{2}-y^{2}}$ orbitals produces the orbital states $d_{3 x^{2}-r^{2}}$ and $d_{3 y^{2}-r^{2}}$, which alternate on the Mn sites in the $a b(x y)$ plane, as shown in Figure 5c. In this case, the Jahn-Teller distortion produces a macroscopic lattice strain, compressing the $c$ axis and expanding the $a b$

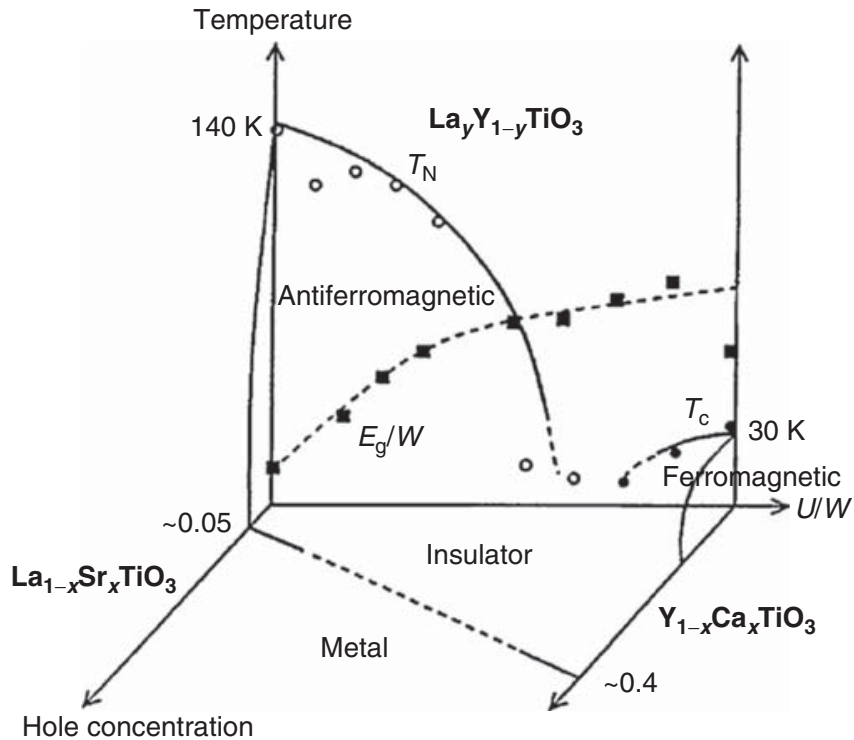

Figure 3. Metal-insulator phase diagram of $\mathrm{RE}_{1-x} \mathrm{AE}_{x} \mathrm{TiO}_{3}$. In the end plane (temperature vs. electron correlation $U M$ ) with $x=0$, the antiferromagnetic $\left(T_{N}\right)$ and ferromagnetic $\left(T_{c}\right)$ transition temperatures are plotted for the $R E=L_{y} Y_{1-y}$ system, where the variation of the normalized Mott-Hubbard energy gap $\left(E_{\mathrm{g}} / W\right)$ is also shown.

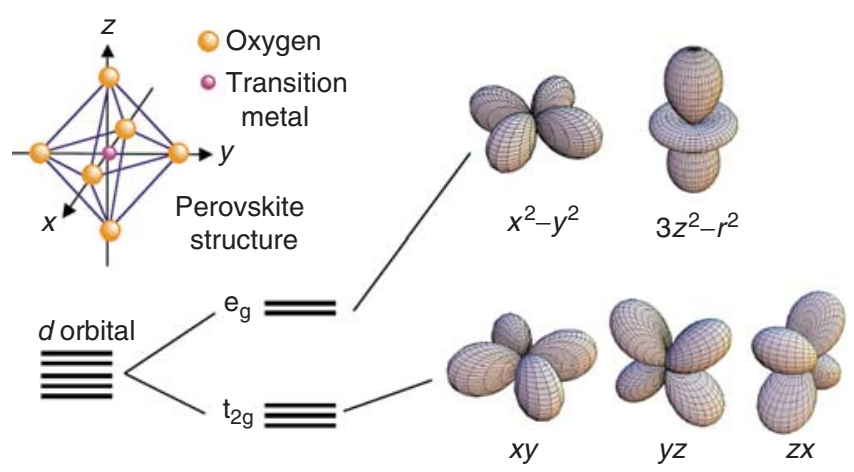

Figure 4. $d$-electron orbitals and level splitting in the octahedral coordination of $\mathrm{O}^{2-}$.

plane. The spins couple ferromagnetically on the $a b$ plane, whereas they are stacked antiferromagnetically along the $c$ axis, producing the layered antiferromagnetic state. ${ }^{10}$ In $\mathrm{BiMnO}_{3}$, with a lower crystal symmetry because of the lone pair on $\mathrm{Bi}^{3+}$ (Figure 5d), the complex orbital order can give rise to a Mott insulating ferromagnetic ground state. ${ }^{11}$

In some relatively common cases, the compound remains electrically insulating or marginally metallic over a broad range of band fillings, in which a periodic array of doped holes or electrons appears. This phenomenon is called charge ordering. Figure 6 exemplifies several cases of such charge ordering in some quasi-two- dimensional transition-metal $(M)$ oxides (RE, $\mathrm{AE})_{2} \mathrm{MO}_{4}$, with the $\mathrm{K}_{2} \mathrm{NiF}_{4}$-type structure (Figure 6a). In the isolated MO sheet of this crystal form, the spin, charge, and/or orbital tend to take the form of stripes. In $\mathrm{La}_{2-x} \mathrm{Sr}_{x} \mathrm{NiO}_{4}$ (Figure 6a, with $\mathrm{RE}=\mathrm{La}$ and $\mathrm{AE}=\mathrm{Sr}$ ), for example, the hole doping, $x$, in the parent Mott insulator $\mathrm{La}_{2} \mathrm{NiO}_{4}$ cannot induce the insulator-metal transition up to $x=0.9$ and instead forms a charge (hole) and spin stripe pattern running parallel to the diagonal direction of the $\mathrm{NiO}_{4}$ squares ("diagonal stripe"), that is, along [110] in the tetragonal lattice. ${ }^{12}$ At $x=1 / 3$, in particular, the charge and orbital stripe state is the most stable, as shown in Figure 6b. 
A similar hole stripe is also known to exist in some of the high-temperature superconducting copper oxides, as exemplified in Figure $6 c$ for the $x=1 / 8$ holedoped $\mathrm{La}_{2} \mathrm{CuO}_{4} \cdot{ }^{13,14}$ In this case, the one-quarter-filled (i.e., $50 \%$ hole-doped) stripes run along the [100] or [010] direction ("vertical stripe") in the half-filled $\mathrm{CuO}_{2}$ background. The incommensurate spin order observed in the underdoped region (say, $x<0.12$ ) of $\mathrm{La}_{2} \mathrm{CuO}_{4}$ might originate from this charge order. Reflecting the one-quarter-filled nature of the vertical stripe, the electrical conduction along the stripe appears to survive and manifests itself as a one-dimensional charge dynamics and Fermi-surface structure.
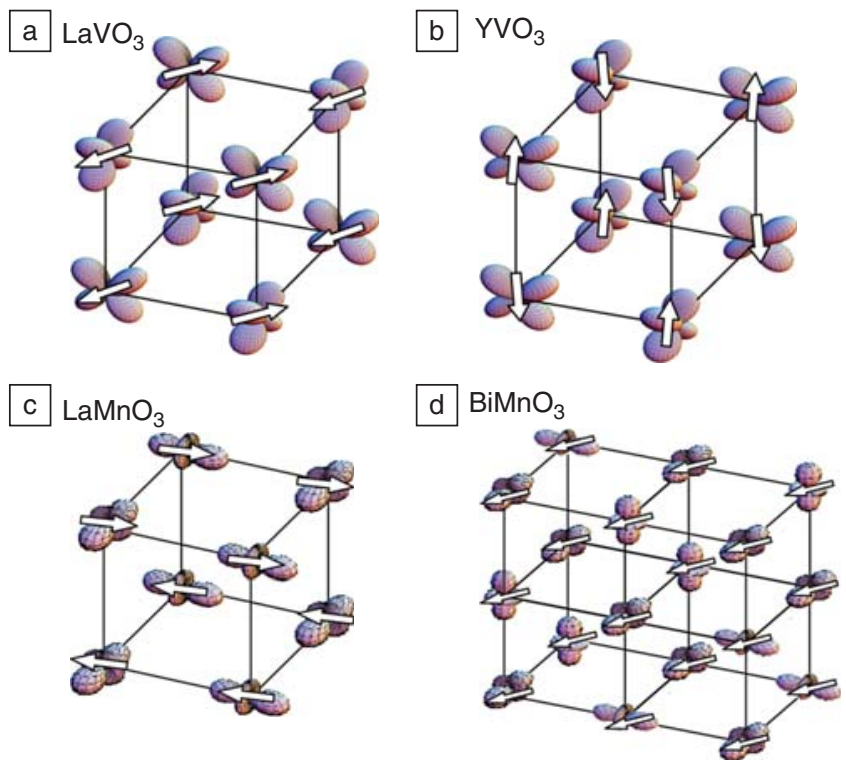

Figure 5. Spin-charge ordering of $d$ electrons in (a) $\mathrm{LaVO}_{3}$, (b) $\mathrm{YVO}_{3}$, (c) $\mathrm{LaMnO}_{3}$, and (d) $\mathrm{BiMnO}_{3}$, all with (distorted) perovskite structures.

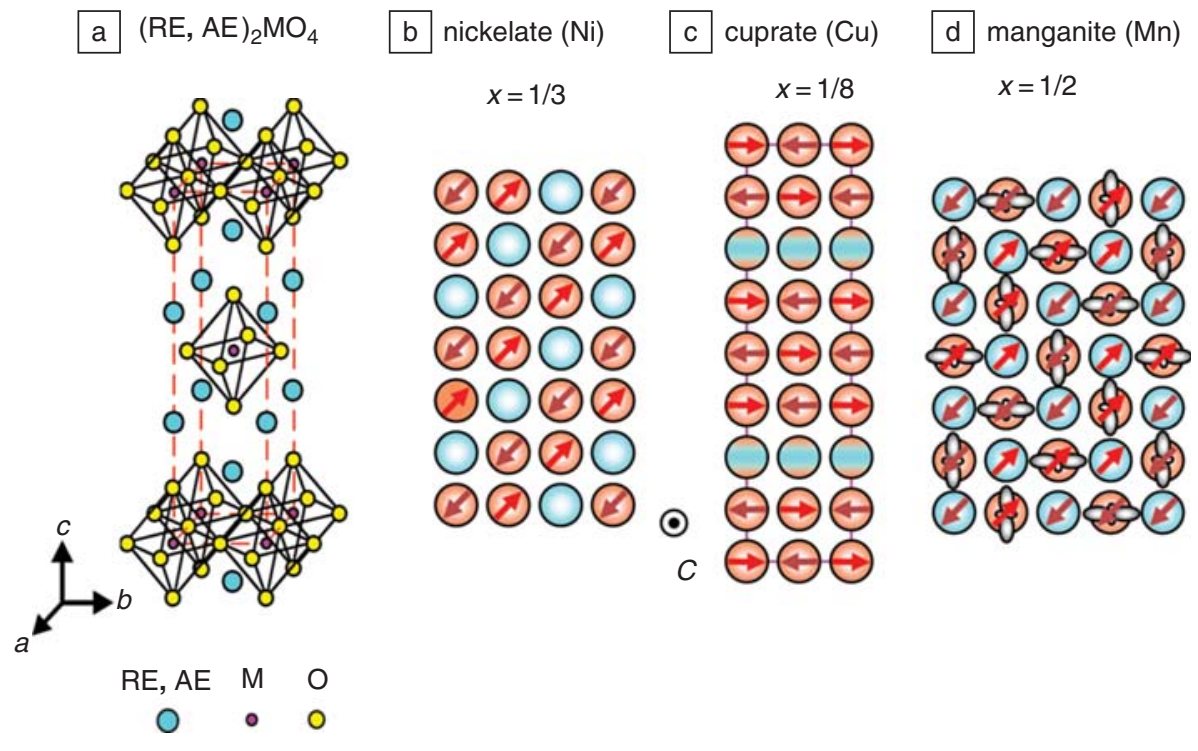

Figure 6. Charge order in the variety form of stripes in hole-doped two-dimensional metal oxide sheets of layered perovskite materials: (a) $\mathrm{K}_{2} \mathrm{NiF}_{4}$-type structure $\left[(\mathrm{RE}, \mathrm{AE})_{2} \mathrm{MO}_{4}\right.$, where $\mathrm{RE}, \mathrm{AE}$, and $\mathrm{M}$ represent the trivalent rare-earth and divalent alkaline-earth ions and the transition-metal element, respectively], (b) $\mathrm{La}_{2-x} \mathrm{Sr}_{x} \mathrm{NiO}_{4}(x=1 / 3)$, (c) $\mathrm{La}_{2-x} \mathrm{Ba}_{x} \mathrm{CuO}_{4}$ $(x=1 / 8)$, and (d) $\mathrm{La}_{1-x} \mathrm{Sr}_{1+x} \mathrm{MnO}_{4}(x=1 / 2)$.
Even more complex features arise in the doped manganites as a consequence of the close interplay among the spin, charge, orbital, and lattice degrees of freedom, as exemplified in Figure $6 \mathrm{~d}$ for a hole-doping levels of $x=1 / 2 .{ }^{15}$ In a classical picture, $\mathrm{Mn}^{4+}$ (with three $\mathrm{t}_{2 \mathrm{~g}}$ electrons as the local $S=3 / 2$ spin) and $\mathrm{Mn}^{3+}$ (with the $S=3 / 2$ local spin plus one $\mathrm{e}_{\mathrm{g}}$ electron) should coexist. The charge ordering shows a checkerboard pattern, whereas the orbital on the same $\mathrm{Mn}^{3+}$ sites shows the larger unit-cell ordering with alternating $d_{3 x^{2}-r^{2}}-$ $d_{3 y^{2}-r^{2}}\left(\right.$ or $\left.d_{y^{2}-z^{2}}-d_{z^{2}-x^{2}}\right)$ orbital occupancy. The observed spin ordering pattern is the compromise between the antiferromagnetic superexchange interaction among the $t_{2 g}$ local spins and the ferromagnetic doubleexchange interaction mediated by the $e_{g}$ electron hopping between the $\mathrm{Mn}^{3+}$ and $\mathrm{Mn}^{4+}$ sites. The orbital ordering regulates the anisotropic $e_{g}$ electron hopping, and hence, the $\mathrm{Mn}^{4+}$ sites adjacent to the lobe of the $\mathrm{e}_{\mathrm{g}}$ orbital on the nearest $\mathrm{Mn}^{3+}$ site is linked through a ferromagnetic interaction. As a result, ferromagnetic zigzag chains appear along the diagonal direction in the ground state. These complex forms of charge-orbital-spin ordering are ubiquitous in the highly (moderately) doped, nonmetallic manganese oxides with perovskite-related structure. Incidentally, the metallic counterpart of the doped manganites is viewed as the orbitals quantum-disordered state with nearly isotropic ferromagnetic double-exchange interactions.

\section{Giant Responses in Correlated- Electron Systems}

The several competing tendencies in correlated-electron materials lead to rich phase diagrams, containing a variety of phases with spin, charge, and orbital order; superconducting states; metals and insulators; multiferroics; and other phases (Figure 7). These phases are interesting in themselves, but in addition, their presence in the same material suggests that their energies must be similar, given that small changes in composition, temperature, pressure, external fields, and other parameters tend to induce a transition from one to another. These transitions are often of first order, which is natural for states with such different properties. However, often, these transitions are more complex than merely involving an abrupt change from one state to another when some parameter is varied.

In many materials, giant responses to external fields have been observed in the region of phase competition. A typical and widely studied example is the case of the colossal magnetoresistance (CMR) in 
manganites. ${ }^{17,18}$ At particular chemical compositions, these materials present a ferromagnetic metallic phase, but with exotic resistivity versus temperature curves. The resistivity is insulating above the ferromagnetic ordering temperature $T_{c}$, it presents a sharp peak at $T_{C}$ upon cooling, and finally it drops to a metallic state as the temperature is lowered further. This curve is exotic because it is unusual to have an insulator as the "normal" state of a low-temperature metallic ferromagnet. However, the most curious property is obtained by introducing an external magnetic field of only a few teslas. In this case, the resistivity peak is rapidly suppressed, generating a large and negative magnetoresistance effect, which was called colossal magnetoresistance in the early days of its discovery in 1994. Although technological applications of this effect in read sensors will need $T_{c}$ to be raised by a substantial factor of two to three, the CMR phenomenon defines a fundamental science puzzle that many researchers have been trying to decipher in recent years.

The present understanding of the CMR effect is based on phase competition. In fact, of the several manganites obtained by varying the trivalent and divalent ions in the chemical formula, only those with relatively small bandwidths show the CMR effect. Concomitant with a small bandwidth, competing insulating states are often found in the phase diagrams. A small bandwidth enhances the relative importance of electron-phonon couplings, and complex charge, orbital, and spin order arrangements are found at low temperatures.

Theoretically, it was conjectured early on that the region of phase competition between the ferromagnetic metal and the insulator is where CMR is found. ${ }^{18}$ This hypothesis was confirmed by recent stateof-the-art computer simulations that use the double-exchange model for manganites, supplemented by a robust coupling to lattice displacements. ${ }^{19,20}$ Some representative theoretical curves are shown in Figure 8. It is clear that these results, which were obtained using Monte Carlo simulations employing large clusters of computers, present resistivity curves that resemble those obtained in experiments. It can be said that the CMR effect is "trapped in a box," and it is up to materials scientists to ask the proper questions to the computers to understand its origin more deeply.

Investigations suggest ${ }^{19}$ that, in the insulating region above $T_{C}$, the shortdistance charge and spin order closely resemble that of the insulating state that

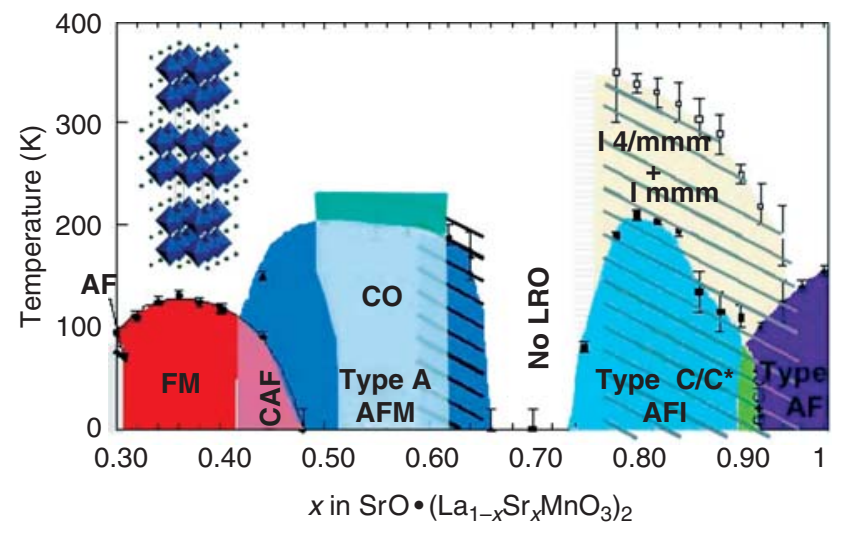

Figure 7. Phase diagram of a typical strongly correlated transition-metal oxide, illustrating the many phases found by varying the chemical composition and temperature. This result corresponds to a bilayer manganite, and it is adapted from Reference 16, where the properties of the many phases and other details can be found. Note: AF is antiferromagnetic, AFI is antiferromagnetic insulator, AFM is antiferromagnetic metallic, CAF is canted antiferromagnetic insulator, $\mathrm{CO}$ is charge-ordered, FM is ferromagnetic, and LRO is long-range order.
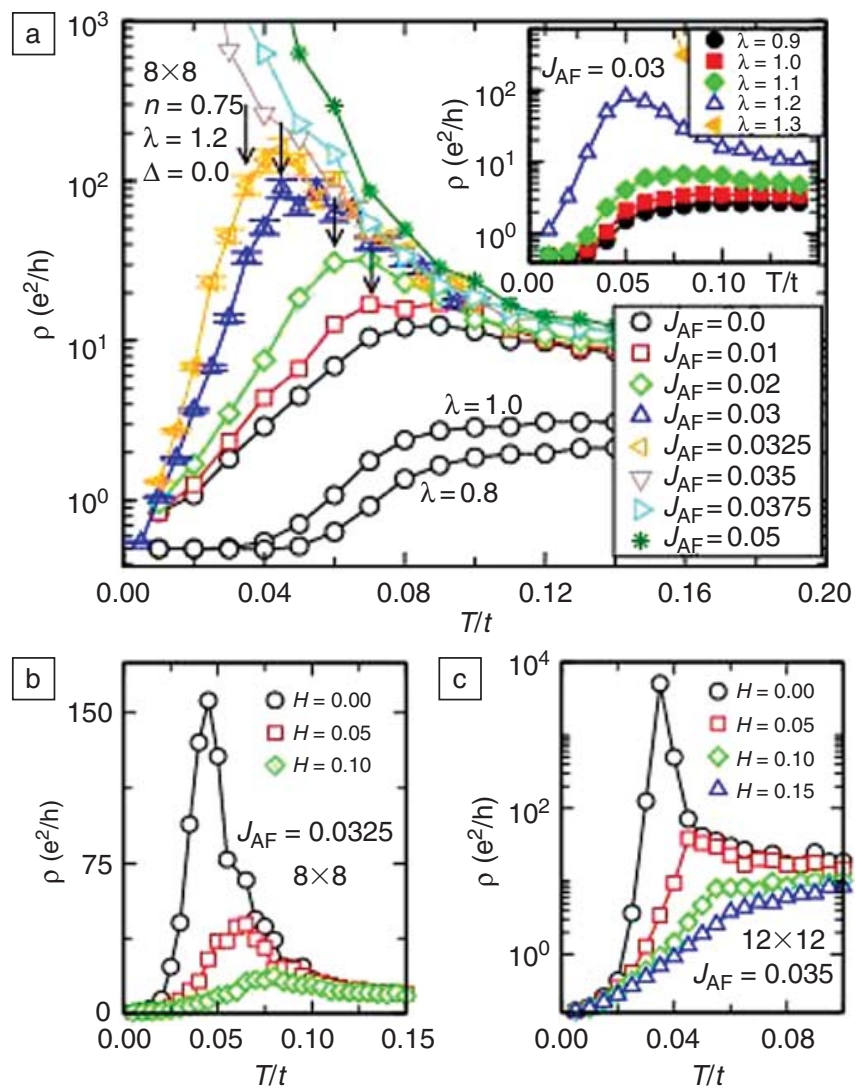

Figure 8. Results of a computer simulation for a model for manganites, adapted from Reference 19. Shown are the resistivity $(\rho)$ versus temperature $(T / t$, where $t$ is the electron hopping amplitude) curves, parametric with couplings in the model, described in more detail in the original publication. The characteristic resistivity peak of materials exhibiting colossal magnetoresistance (CMR) is observed, particularly in (a). In (b) and (c), the rapid reduction of the resistance with increasing magnetic field $H$ is shown. Note: $J_{A F}$ is the antiferromagnetic coupling constant, $n$ is the band filling, $\lambda$ is the electron phonon coupling, $e$ is the charge of the electron, $\mathrm{h}$ is planck's constant, and $\Delta$ is the strength of the quenched disorder. 
competes with ferromagnetism. Perhaps induced by the dominance of a large entropy above $T_{c}$, the system behaves as if the insulator were the dominant state. However, at $T_{c}$, the energy prevails over the entropy, and the true metallic state is stabilized upon cooling. Moreover, it has been observed, ${ }^{19-21}$ both theoretically and experimentally, that quenched disorder caused by chemical doping enhances the window in parameter space where these effects are observed. A schematic phase diagram including both bandwidth and disorder strength was predicted by theory and confirmed by experiments. In this phase diagram, shown in Figure 9, an original first-order transition is smeared by disorder, and it is in the intermediate region, which has glassy properties, where the colossal effect is observed. ${ }^{18,21}$

The giant nonlinearity observed in CMR manganites originating in phase competition is simply one example of the potentially enormous responses that many correlated-electron systems could have. In fact, the sketch in Figure 9 does not depend in any detail on the properties of the competing states, but rather depends on the competition itself. Note, however, that, if one of the phases is not ferromagnetic, then the external perturbation that triggers the giant response might be less obvious than a magnetic field. In fact, recent investigations have suggested giant responses in underdoped cuprates. In this case, the external perturbation is the proximity of a well-developed superconductor that induces superconductivity in a non-superconducting underdoped cuprate. ${ }^{22,23}$ More generally, it might be that the exotic properties of the famous pseudogap regime of the high-temperature superconductors originates in phase competition between the parent compound, which is an antiferromagnetic insulator, and the superconducting state itself. In addition, many other correlated-electron materials might have similar exotic nonlinear properties. Surprises of this kind might be found in several compounds of the correlated-electron family.

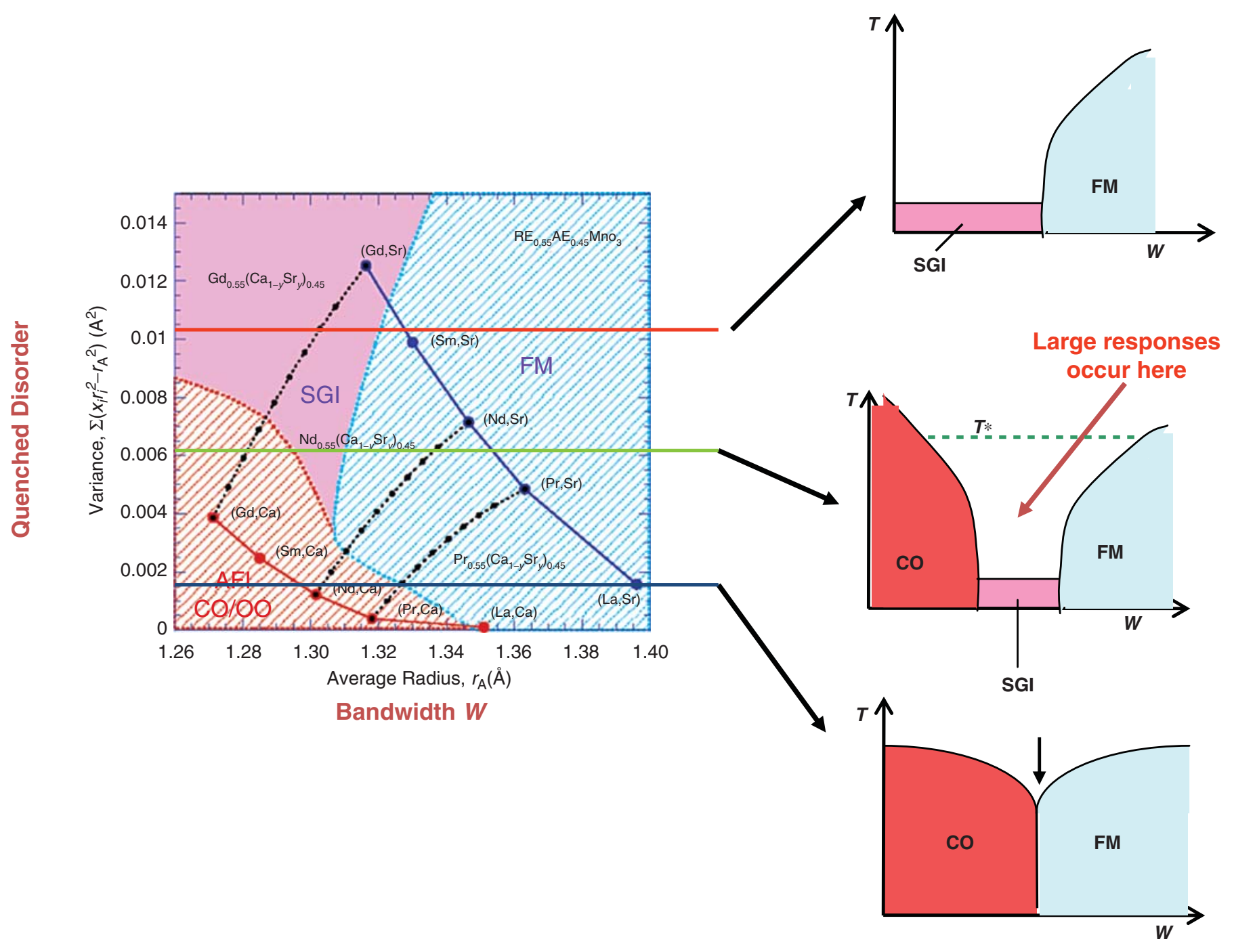

Figure 9. Generic phase diagram of manganites with varying bandwidth and quenched disorder. At a low degree of quenched disorder, a firstorder transition separates the competing ferromagnetic (FM) metallic and charge-ordered (CO) states. As this disorder strength increases, a window opens between the competing states, and a glassy region with nanoscale inhomogeneities is formed. The large response to magnetic fields (colossal magnetoresistance, or CMR) appears to occur in this regime, near the (now-reduced) Curie temperature. A new crossover temperature $T^{*}>T_{\mathrm{C}}$ is observed in this regime, where the nanoscale clusters start forming. Finally, at large values of the disorder strength, even the $\mathrm{CO}$ state is no longer observed. Note: SGI is spin-glass insulator. 
Note that the nonlinearities described herein appear to occur concomitantly with nanometer-length-scale inhomogeneities. ${ }^{18,24}$ A variety of experiments in manganites have convincingly shown that, in the CMR regime, there are nanoscopic clusters with characteristics similar to those of the charge-ordered state that is stable at half-doping, even though the ground state is a ferromagnetic metal. Even before the experimental investigations in this context, theory had predicted strong tendencies toward inhomogeneous states, a tendency widely known as phase separation. ${ }^{25}$ Self-organization of the electronic degrees of freedom also occurs in some high-temperature superconductors and nickelates where states with stripes have been identified, as previously described. ${ }^{14}$ Recent experiments ${ }^{26}$ have shown that other types of inhomogeneous states occur in the superconducting cuprates (Figure 10).

\section{Outlook for Correlated-Electron Technology}

The use of both spin and charge degrees of freedom is an essential feature for the emerging field of spin-electronics, or spintronics. Its straightforward application is the control of the electrical current by an external magnetic field. In fact, the inventions of a giant-magnetoresistive magnetic multilayer (composed of transition metals) and a tunneling magnetoresistance (TMR) device were crucial milestones for the field of spintronics and its industrial application.

For the general spintronic use, a halfmetal, meaning a metallic ferromagnet with perfect spin polarization in the ground state, is of great interest. Several of the ferromagnetic metallic transitionmetal oxides with strong electron correlation are expected to show such a half-metallic ground state because of potentially strong spin-charge coupling. Hole-doped manganites with a perovskite structure, $\mathrm{La}_{1-x} \mathrm{Sr}_{x} \mathrm{MnO}_{3}(0.2<x<0.5)$, provide a typical example of a half-metal mediated by the strong Hund coupling between the $e_{g}$ electron spin and $t_{2 g}$ local spins. However, the TMR characteristics for the junction that uses $\mathrm{La}_{1-x} \mathrm{Sr}_{x} \mathrm{MnO}_{3}$ are known to show an unexpected degradation with increasing temperature, say, up to $200 \mathrm{~K}$, despite their higher Curie temperatures (330-370 K). Consensus has now been reached that this seemingly rapid fading out of the spin polarization is due to the modification of the interface magnetism of $\mathrm{La}_{1-x} \mathrm{Sr}_{x} \mathrm{MnO}_{3}(0.2<x<0.5)$ when facing the insulating barrier. To attack this problem, alternative engineered magnetic interfaces are being

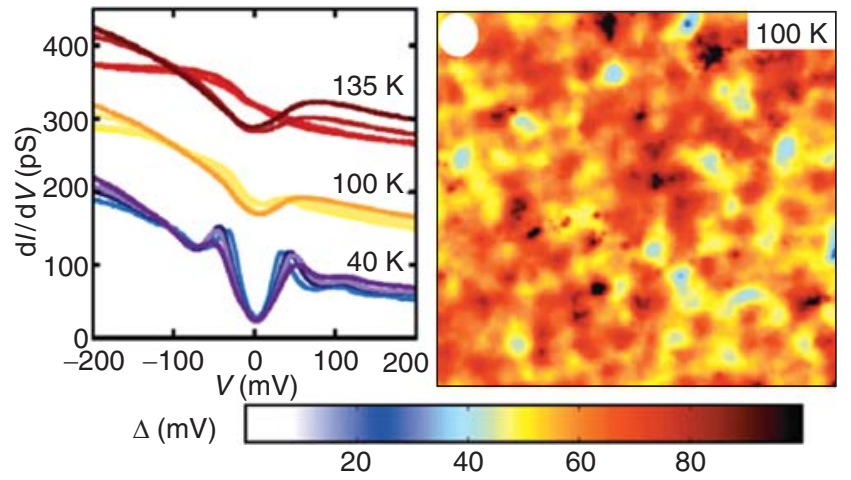

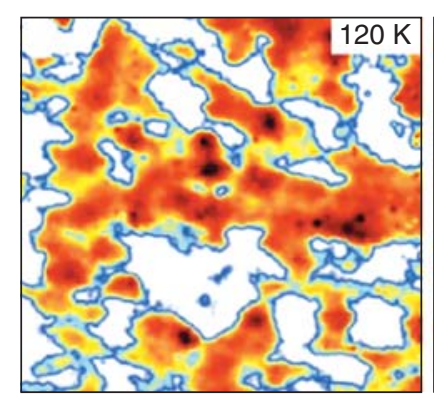

$T(\mathrm{~K})$

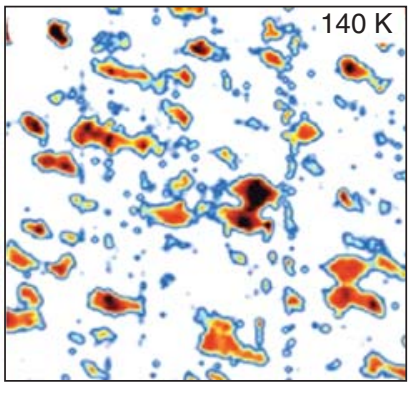

$T(\mathrm{~K})$

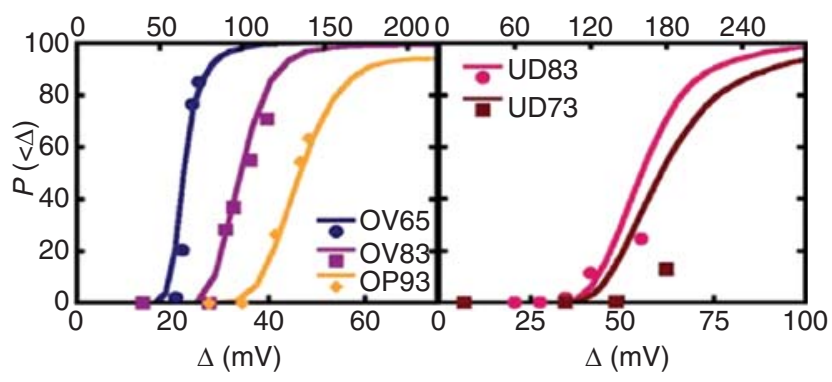

Figure 10. Example of an experimental result showing inhomogeneous behavior in a transition-metal oxide. Shown are results reported in Reference 26, containing scanning tunneling microscopy (STM) data gathered using a high-temperature superconductor with a critical (optimal) temperature of $93 \mathrm{~K}$. The colored regions have a $d$-wave STM spectrum, and the remarkable result is that these superconducting areas are observed even at temperatures as high as $120 \mathrm{~K} . \Delta$ is the superconducting gap obtained from the local density of states measured with the STM technique.

extensively explored. ${ }^{27}$ Moreover, the emerging novel properties and possible functionalities at the interfaces between correlated oxides represent a new and important area of investigations..$^{28-31}$

More robust and higher- $T_{\mathrm{c}}$ half-metals have also been sought in the family of perovskites. A consequence of such investigations was the discovery of the ordered double perovskite family with half-metallic characteristics, represented by $\mathrm{Sr}_{2} \mathrm{~B}_{1}$ $\mathrm{B}_{2} \mathrm{O}_{6}\left(\mathrm{~B}_{1}=\mathrm{Fe}\right.$ or $\mathrm{Cr}, \mathrm{B}_{2}=$ Mo or Re $) \cdot{ }^{32}$ In this class of compounds, the perovskite $B$ (transition-metal) sites are alternately occupied by $B_{1}$ and $B_{2}$ in a rock-salt form. The valence of $\mathrm{Fe}$ (or $\mathrm{Cr}$ ) is $3+$, corresponding to the maximum spin state $S=5 / 2(3 / 2)$. The $\mathrm{Mo}^{5+}\left(4 d^{1}\right)$ or $\mathrm{Re}^{5+}\left(5 d^{2}\right)$ ion provides the conduction electrons, partially hybridizing with the spin-down state of Fe or $\mathrm{Cr}$, and strongly coupling antiferromagnetically with the local spins (spin-up states) on Fe or Cr. Thus, the states near the Fermi level are composed only of the spindown electrons, forming the half-metallic ground state. The ferromagnetic transition temperature can be very high, for example, $420 \mathrm{~K}$ for $\mathrm{Sr}_{2} \mathrm{FeMoO}_{6}$ and as high as $615 \mathrm{~K}$ for $\mathrm{Sr}_{2} \mathrm{CrReO}_{6}$. Possible antiferromagnetic metals with a half-metallic ground state, which is a highly spin-polarized state without any magnetization, have also been sought for in the family of ordered perovskites, but so far, the attempt has not been successful because of the correlationinduced charge-gap opening. 
Another important group of compounds related to spintronic oxides is the multiferroics, that is, materials with coexisting ferroelectric and magnetic orders, which can potentially host a gigantic magnetoelectric (ME) effect. Concerning the coupling between the magnetism and the ferroelectricity, a new simple scheme has recently been theoretically 33,34 and experimentally ${ }^{35}$ demonstrated; the mutually canted spins (Figure 11a), as generated by the spin frustration effect, can produce an electronic polarization through the spin-orbit interaction. When the spins form a transverse-spiral (cycloidal) modulation along the specific crystallographic direction (Figure 11b), then every nearestneighbor pair produces a unidirectional polarization $p_{i}$, and, hence, a macroscopic polarization $P$ of electronic origin should be generated. The direction of the polarization can be completely determined by the clockwise or counterclockwise rotation of the spins that are proceeding along the spiral propagation axis, called the spin helicity.

In these cycloidal spin compounds, typically perovskite $\mathrm{TbMnO}_{3}$ and $\mathrm{DyMnO}_{3}$, the spontaneous polarization can be easily controlled by an external magnetic field of a specific direction, inducing the generation and/or flipping of the spontaneous polarization, ${ }^{35}$ which can be viewed as a gigantic ME effect. Multiferroics based on this mechanism have also been realized in the conical spin state (Figure 11c) where the transverse spiral component coexists with the uniform (spontaneous or fieldinduced) magnetization component along the cone axis, and hence, the flexible control of the polarization vector is possible by external magnetic field. ${ }^{36}$ These cycloidal and transverse-conical spin states are widely seen in complex transition-metal compounds such as spinels and perovskites, where competing exchange interactions of the neighboring spins can cause such a periodically modulated spin structure. As the natural extension of these studies, the electrical control of the magnetization vector is now being explored as a new and potentially important spintronic function.

In analogy to spintronics, one can also consider the possibility of controlling electric currents through the $d$-electron orbital state. ${ }^{37}$ Here, we call this possible correlatedelectron technology orbital-electronics or orbitronics. In a broad context, the CMR phenomenon itself amounts to a fieldinduced modification of the orbital correlation, and hence, it could provide one such example. By analogy to the magnetoresistance effect in the spin-ordered state, one can utilize the orbital degree of

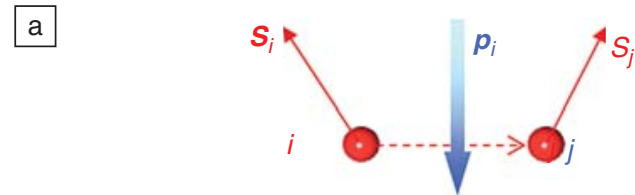

$\mathrm{b}$
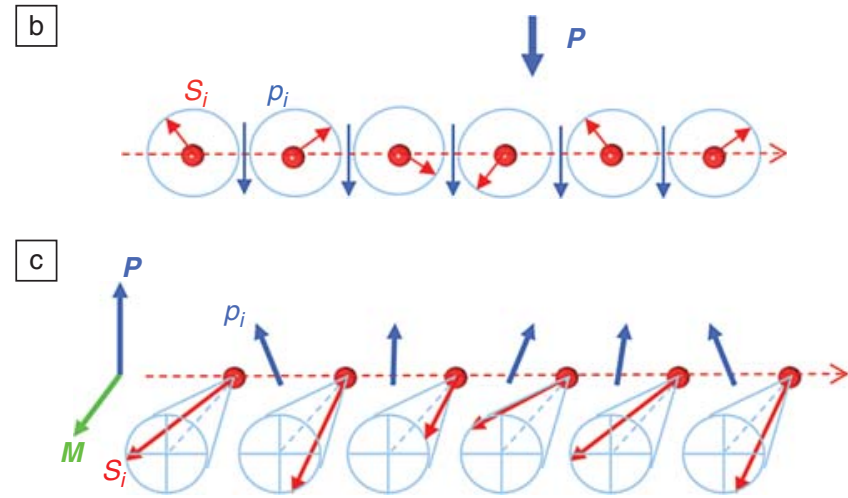

Figure 11. Possible spin superstructures in multiferroics with strong coupling between the magnetism and electricity. (a) Canted spins $\left(S_{i}, S_{i}\right)$ on neighboring atomic sites. (b) Spin spiral structure producing a uniform polarization $(P)$. (c) Conical spin structure allowing both uniform magnetization $(M)$ and $P$.

freedom to regulate the electrical conduction, as shown in Figure 12a. For example, when the orbital ordering composed of $d_{x_{2}-y_{2}}$ orbitals is realized in the nearly cubic perovskite lattice, the charge dynamic is highly anisotropic, specifically, confined within the $x y$ plane and insulating along the $z$ direction, as can be noticed from the transfer hopping values shown in Figure 12a. In reality, such a ferroic orbital ordering is present ubiquitously in overdoped manganites, and a highly two-dimensional charge motion is observed despite the nearly cubic lattice structure.

The key idea of orbitronics is the ultrafast switching of the orbital state, and hence of the related spin-charge state, by means of electric fields and/or light irradiation. Because the orbital shape, rodlike or planar, represents the electrons probability-density distribution, the orbital degree of freedom can inherently couple with the electric field through its anisotropic polarizability. In this context, the orbital manipulation might bear an analogy to liquid-crystal technology, in which rod-shaped or planar molecules can respond to the electric field through their anisotropic polarizability.

To describe the dynamical response of the orbital to the external fields, we need to define the orbital wave or orbiton in the orbital-ordered state (Figure 12b). An advantage of the use of the orbital degree of freedom in ultrafast control of the electronic and magnetic state is that the orbiton frequency is high, ${ }^{38} 10-100 \mathrm{THz}$,

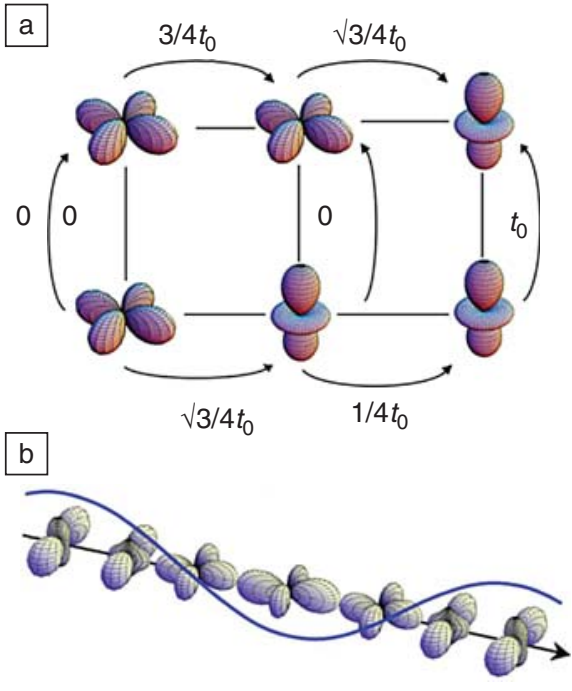

Figure 12. (a) Electron hopping amplitudes between the nearestneighbor sites with various orbital states. $t_{0}$ stands for the transfer integral unit. (b) Quantal orbital wave, or orbiton, of the orbital-ordered state.

as compared to the typical spin precession frequency (i.e., the $\mathbf{k}=0$ magnon energy in a ferromagnet) of 1-100 GHz. In fact, in the initial process of the photoinduced insulator-metal transition in the perovskite manganite $\left(\operatorname{Pr}_{07} \mathrm{Ca}_{03} \mathrm{MnO}_{3}\right)$, the orbiton mode of $30 \mathrm{THz}$ is observed in ultrafast optical spectroscopy. ${ }^{39}$ 
Correlated-electron science is thus exploiting a broad range of materials and electronic properties, in addition to the famous high- $T_{c}$ and CMR-related phenomena that have received so much attention. Noteworthy is the recent advance of the epitaxial-growth technology for transition-metal oxide thin films and superlattices, as well as the discoveries of many intriguing properties at the heterointerfaces. At the interface, the spin and orbital, as well as charge, states are greatly modified, and they can be subject to external magnetic and/or electric fields that can induce large nonlinear effects. One of the potential applications of such phenomena is the resistance switching memory effect of the correlated-oxide interface with metal electrodes; the mechanisms are still to be clarified, but the possible application of this effect to high-density, fast, nonvolatile memory devices (resistive random-access memory, or ReRAM) is now being investigated extensively (see, for example, Reference 40). Another interesting example is the creation of a polar ferromagnet with broken inversion symmetry at the interface, in which many new intriguing magnetoelectronic functions, such as nonlinear/ nonreciprocal magneto-optical and dynamical magnetoelectric effects, can be observed because of the simultaneous breaking of space-inversion and timereversal symmetries.

\section{Conclusions}

The study of correlated electronic materials continues at a fast pace. A close interaction between theory and experiments has been crucial for the progress in this field. The complexity of these compounds, manifested in their rich phase diagrams, self-organization, and nonlinear responses, suggests their potential use in devices. Yet, even if technological applications take considerable time to be realized, the interesting properties of these materials define a field of fundamental scientific research that is full of surprises and will surely continue to provide exciting and challenging phenomena in the near future.

\section{References}

1. Y. Tokura, N. Nagaosa, Science 288, 462 (2000).

2. E. Dagotto, Science 309, 257 (2005).

3. Y. Kamihara, T. Watanabe, M. Hirano,

H. Hosono, J. Am. Chem. Soc. 130, 3296 (2008).

4. Committee on CMMP 2010, CondensedMatter and Materials Physics: The Science of the World Around Us (National Academies Press, Washington, DC, 2007).

5. Basic Energy Sciences Advisory Committee (BESAC) Grand Challenges Subcommittee, Directing Matter and Energy: Five Challenges for Science and the Imagination, (U.S. Department of Energy, Washington, DC, 2007).

6. M. Imada, A. Fujimori, Y. Tokura, Rev. Mod. Phys. 70, 1039 (1998).

7. J.B. Torrance, P. Lacorre, A.I. Nazzal, E.J. Ansaldo, Ch. Niedermayer, Phys. Rev. B 45, 8209 (1992).

8. Y. Tokura, Y. Taguchi, Y. Fujishima, T. Arima, K. Kumagai, Y. Iye, Phys. Rev. Lett. 70, 2126 (1993). 9. H. Sawada, N. Hamada, K. Terakura, K.T. Asada, Phys. Rev. B 53, 12742 (1996).

10. J. Kanamori, J. Phys. Chem. Solids 10, 87 (1959).

11. A. Moreira dos Santos, A.K. Cheetham, T. Atou, Y. Shono, Y. Yamaguchi, K. Ohyama, H. Chiba, C.N.R. Rao, Phys. Rev. B 66, 064425 (2002).

12. C.H. Chen, S.-W. Cheong, A.S. Cooper, Phys. Rev. Lett. 71, 2461 (1993).

13. J.M. Tranquada, B.J. Sternlieb, J.D. Axe, Y. Nakamura, S. Uchida, Nature 375, 561 (1995)

14. S.A. Kivelson, E. Fradkin, V.J. Emery, Nature 393, 550 (1998).

15. Y. Murakami, H. Kawada, H. Kawata, M. Tanaka, T. Arima, Y. Moritomo, Y. Tokura, Phys. Rev. Lett. 80, 1932 (1998).

16. J.F. Mitchell, D.N. Argyriou, A. Berger, K.E. Gray, R. Osborn, U. Welp, J. Phys. Chem. B 105, 10731 (2001)

17. S. Jin, T.H. Tiefel, M. McCormack, R.A Fastnacht, R. Ramesh, L.H. Chen, Science 264, 413 (1994)

18. E. Dagotto, T. Hotta, A. Moreo, Phys. Rep. 344, 1 (2001).
19. C. Sen, G. Alvarez, E. Dagotto, Phys. Rev. Lett. 98, 127202 (2007) and references therein. 20. S. Kumar, P. Majumdar, Phys. Rev. Lett. 96, 016602 (2006)

21. D. Akahoshi, M. Uchida, Y. Tomioka, T. Arima, Y. Matsui, Y. Tokura, Phys. Rev. Lett. 90, 177203 (2003).

22. G. Alvarez, M. Mayr, A. Moreo, E. Dagotto, Phys. Rev. B 71, 014514 (2005).

23. G. Alvarez, E. Dagotto, arXiv:0802.3394, to appear in Phys. Rev. Lett.

24. M. Uehara, S. Mori, C.H. Chen, S.-W. Cheong, Nature 399, 560 (1999).

25. S. Yunoki, J. Hu, A.L. Malvezzi, A. Moreo, N. Furukawa, E. Dagotto, Phys. Rev. Lett. 80, 845 (1998).

26. K. Gomes, A.N. Pasupathy, A. Pushp, S. Ono, Y. Ando, A. Yazdani, Nature 447, 569 (2007).

27. H. Yamada, Y. Ogawa, Y. Ishii, H. Sato, M. Kawasaki, H. Akoh, Y. Tokura Science 305, 646 (2004).

28. A. Ohtomo, H.Y. Hwang, Nature 427, 423 (2004).

29. N. Reyren, S. Thiel, A.D. Caviglia, L. Fitting Kourkoutis, G. Hammerl, C. Richter, C.W. Schneider, T. Kopp, A.-S. Rüetschi, D. Jaccard, M. Gabay, D.A. Muller, J.-M. Triscone, J. Mannhart, Science 317, 1196 (2007).

30. S. Okamoto, A. Millis, Nature 428, 630 (2004).

31. E. Dagotto, Science 318, 1076 (2007).

32. K.I. Kobayashi, T. Kimura, H. Sawada, K. Terakura, Y. Tokura, Nature 395, 677 (1998).

33. H. Katsura, N. Nagaosa, A.V. Balatsky, Phys. Rev. Lett. 95, 057205 (2005).

34. I.A. Sergienko, E. Dagotto, Phys. Rev. B 73, 094434 (2006).

35. T. Kimura, T. Goto, H. Sintani, K. Ishizaka, T. Arima, Y. Tokura, Nature 426, 55 (2003).

36. S. Ishiwata, Y. Taguchi, H. Murakawa, Y. Onose, Y. Tokura, Science 319, 1643 (2008).

37. Y. Tokura, N. Nagaosa, Science 288, 462 (2000).

38. E. Saitoh, S. Okamoto, K.T. Takahashi, K. Tobe, K. Yamamoto, T. Kimura, S. Ishihara, S. Maekawa, Y. Tokura, Nature 410, 180 (2001).

39. D. Polli, M. Rini, S. Wall, R.W. Schoenlein, Y. Tomioka, Y. Tokura, A. Cavalleri, Nature Materials 6, 643 (2007).

40. S.Q. Lu, N.J. Wu, A. Ignatiev, Appl. Phys. Lett. 76, 2749 (2000).

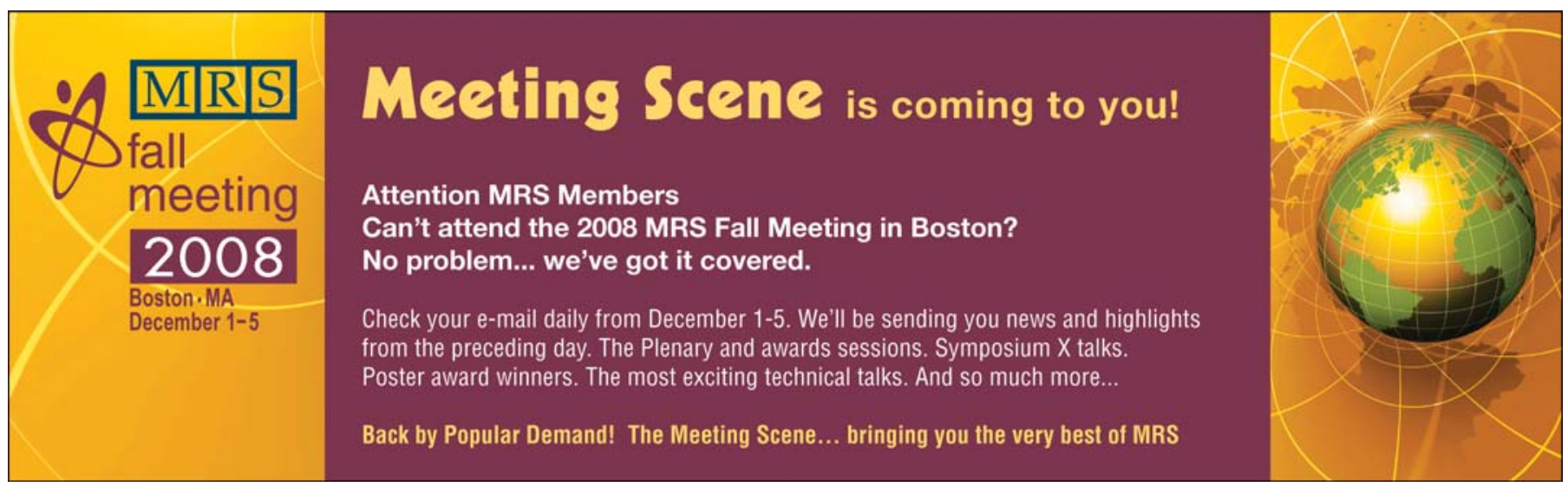

